\title{
Improving Patient-Centredness in Endometriosis Care: A Study Protocol for a Prospective Study with a Mixed-Methods Approach
}

\author{
Anneke M.F. Schreurs ${ }^{a}$ Charlotte I. van Schaijik ${ }^{a}$ Bianca De Bie ${ }^{b}$ \\ Jacques W.M. Maas ${ }^{c, d}$ Cornelis Lambalk ${ }^{a}$ Lisette E.E. van der Houwen ${ }^{a, d}$ \\ Velja Mijatovic a, d \\ aEndometriosis Center, Department of Reproductive Medicine, Research Institute Amsterdam Reproduction and \\ Development, Amsterdam UMC, Vrije Universiteit Amsterdam, Amsterdam, The Netherlands; ${ }^{\text {bEndometriose }}$ \\ Stichting, Dutch Endometriosis Patient Organization, Sittard, The Netherlands; ' Department of Obstetrics and \\ Gynaecology, Maastricht University Medical Center+ and GROW - School for Oncology and Developmental Biology, \\ Maastricht, The Netherlands; ${ }^{d}$ Special Interest Group Endometriosis, Dutch Society of Obstetrics and Gynaecology, \\ Utrecht, The Netherlands
}

\section{Keywords}

Endometriosis - Quality of care - Qualitative research . Improvement of care · Patient-centredness

\begin{abstract}
Objective: Endometriosis is a chronic gynaecologic disease, causing pain and infertility. As there is no definitive cure, patients are subjected to long-term care. This study aimed to improve patient-centred endometriosis care. Patient-centredness of endometriosis care can be evaluated using the validated ENDOCARE questionnaire (ECQ), resulting in centre-specific targets for improvement. To understand how to tackle the targets for improvement as found by the ECQ, focus groups can be organized. Design: This protocol presents a prospective study with a mixed-methods approach to improve patient-centredness of endometriosis care. The study consists of 5 steps: (1) evaluating current patient-centredness of endometriosis care by using the ECQ, (2) understanding targets for improvement, (3) drafting an improvement plan, (4) implementing improvements, and (5) evaluating the improved patient-centredness of endometriosis care.
\end{abstract}

karger@karger.com www.karger.com/goi

Karger"

BOPEN ACCESS
(C) 2021 The Author(s)

Published by S. Karger AG, Basel

This is an Open Access article licensed under the Creative Commons Attribution-NonCommercial-4.0 International License (CC BY-NC) (http://www.karger.com/Services/OpenAccessLicense), applicable to the online version of the article only. Usage and distribution for commercial purposes requires written permission.
The final evaluation will be performed 1.5 years after implementing the improvement plan. Methods: Patient-centredness will be evaluated using the ECQ by inviting women with endometriosis to participate (steps 1 and 5). To investigate step 2, focus groups will be organized. For these focus groups, women with endometriosis are asked to participate until data saturation is achieved. During focus groups, participants are motivated to discuss the found targets for improvement and stimulated to find ways to improve them. The drafting and implementing of the improvement plan (steps 3 and 4) will be organized with the help of health-care providers in close collaboration with the patient organization. To assess whether the implementation of the improvement plan was successful in improving endometriosis care, the results from the ECQ in step 5 will be compared to the results from the ECQ in step 1. Ethical approval was granted by the local Institutional Review Board (Ref 2018.438). Setting: The study was conducted in the university hospital in the Netherlands. Limitations: Both patients and health-care providers will be involved in drafting the improvement plan. By making the health-care providers responsible for improving care, the chance of succeeding is optimized. Whether 
this improvement strategy is successful will be investigated after the implementation of the improvement plan. The improvement plan is clinic specific and can possibly not be extrapolated to other endometriosis clinics. In order to aim to improve patient-centred endometriosis care elsewhere, the complete study protocol should be performed. Conclusions: This study protocol aimed to investigate focus groups as a strategy to identify possible interventions to improve patient-centred endometriosis care by investigating the underlying causes for poor performance on patient-centred care. This study protocol could be used in more endometriosis care centres in the future and might also be useful for improving patient-centredness in other chronic diseases.

(c) 2021 The Author(s).

Published by S. Karger AG, Basel

\section{Introduction}

Endometriosis is a chronic gynaecological disease characterized by symptoms such as dysmenorrhoea, noncyclical pelvic pain, deep dyspareunia, and infertility [1]. In addition, energy loss and fatigue are frequently reported complaints accompanying endometriosis [2]. Therefore, having endometriosis might negatively influence various daily activities as well as productivity $[3,4]$. Furthermore, endometriosis is correlated with an impaired quality of life (QoL) [4-9].

Although different theories have emerged $[10,11]$, the debate on the aetiology of endometriosis continues. In current debates, retrograde menstruation is still regarded as an important origin of the endometrial deposits, but other factors are also involved, including a favourable endocrine and metabolic environment as well as an altered inflammatory response in genetically susceptible women. However, no single theory explains all cases of endometriosis.

Therefore, endometriosis remains a chronic gynaecological disease with no definitive cure. Women with endometriosis need long-term care with frequent check-ups and enduring medical treatment, often provided within a multidisciplinary team of health-care providers. It is therefore important to offer the best care possible which is based on patient-centredness [12].

Patient-centred care is care that "takes into account the preferences and aspirations of individual (health care) service users and the cultures of their communities" [13]. Patient-centredness of endometriosis care can be measured using the ENDOCARE questionnaire (ECQ) [14, 15]. The ECQ is a validated questionnaire that investigates patient-centredness per endometriosis clinic by

Improving Patient-Centredness in

Endometriosis Care: A Study Protocol questioning women with endometriosis on performance and importance of different care aspects associated with patient-centredness. In recent years, this questionnaire has been used in various Dutch-speaking endometriosis patient populations and has shown to be a valuable tool in investigating patient-centredness of endometriosis care $[15,16]$.

One of the results of the ECQ is an overview of strengths and targets for improvement of the different aspects of endometriosis care per endometriosis clinic. However, the ECQ itself does not guide us on how to enhance these targets for improvement. Recently, Geukens et al. [17] suggested that to provide patient-centred endometriosis care, "we should understand their (women with endometriosis) most common preferences, needs, and values." As the targets for improvement, as measured by the ECQ, could be interpreted in various ways, there is need to understand these targets of improvement form a patient's perspective. It is therefore important to explore the preferences, needs, and values of women with endometriosis in relation to the organization of endometriosis care. Only then can targets for improvement be addressed and patient-centredness enhanced. The current study aimed to validate a method to improve endometriosis care by exploring the underlying cause for the targets for improvement of patient-centred endometriosis care and to evaluate the implementation of the improvement plans.

\section{Materials and Methods}

A prospective study with a mixed-methods approach will be performed to study patients' preferences, needs, and values in order to improve patient-centredness of endometriosis care. A mixed-methods approach systematically combines both quantitative and qualitative research methods into 1 single study [18]. The study consists of 5 consecutive steps: (1) evaluating current patient-centredness of endometriosis care by using the validated ECQ, (2) understanding targets for improvement from patients' perspective by using focus groups, (3) drafting an improvement plan in collaboration with health-care professionals and the patient organization, (4) implementing improvements, and (5) evaluating the patient-centredness of endometriosis care, again by performing ECQ evaluations. A flowchart of the study is presented in Figure 1 . The study will be performed in a single Dutch-specialized endometriosis clinic.

\section{Evaluating Current Patient-Centeredness of Endometriosis}

Care

At baseline, the current patient-centredness of endometriosis care will be evaluated by using the ECQ. The ECQ will be distributed among all women diagnosed with endometriosis who consulted the endometriosis clinic in the previous 6 months. Endometriosis can be diagnosed by physical examination, radiologic investigation (includ- 
Step 1:

Evaluating current pa tient centeredness

Duration: 6 months

Involved: women with endometriosis

Step 2:

Understanding targets for improvement

Duration: 3-6 months

Involved: women with endometriosis

Step 3:

Drafting improvement plan

Duration: 3 months

Involved: health care professionals, patient organization
Baseline

measurement:

ENDOCARE

questionnaire

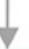

Identifying

targets for

improvement

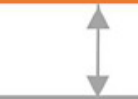

Focus groups

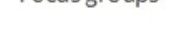

Drafting of

improvement

plan

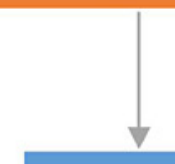

Improvement

plan

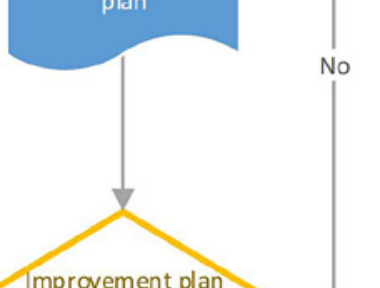

Improvement plan

Sufficient?

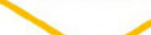

Yes
Fig. 1. Flowchart of study steps. ECQ, ENDOCARE questionnaire.
Step 5:

Evaluating the improved patient centeredness Duration: 6 months

Population: women with endometriosis
Step 4:

Implementing improvement plan

Duration: 1,5 years

Involved: health care professionals

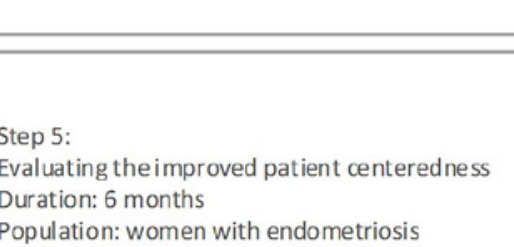

Implementing improvement plan

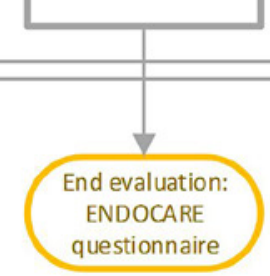


ing transvaginal ultrasound and/or MRI) or surgically, but the gynaecologist has to be certain about the diagnosis.

The ECQ identifies overall patient-centredness scores, but also evaluates performance and importance of 38 aspects of endometriosis care. Performance will be presented in percentage of negative performance (PNP) and importance by mean importance score. By combining care aspects that are rated as important by participants (high mean importance score) and score poor on performance (high PNP), targets for improvement can be identified. The targets for improvement with the highest need for improvement can be identified by calculating quality impact indices $\left(\mathrm{QII}_{\text {care aspect }}=\mathrm{MIS}_{\text {care aspect }} \times \mathrm{PNP}_{\text {care aspect }} / 100\right)[19]$.

\section{Understanding Targets for Improvement}

The goal of step 2 is to understand the underlying cause for specific targets for improvement by exploring women's personal experiences and ideas on the specific targets for improvement. In this part of the investigation, qualitative research methods with a phenomenological approach will be used. A number of reasons led to the decision for using focus groups: (i) the women who participate in the focus groups are not per se the women who reported the specific targets for improvement, making it possibly difficult for them to react to targets for improvement that they personally would not grade as a target for improvement if one would ask them individually, (ii) group discussion will lead to a better overall understanding of the underlying cause, and (iii) gathering different ideas from different women can facilitate a thorough brainstorm session for widely supported ideas for defining targets for improvement.

Focus groups will be organized among women with endometriosis to discuss the targets for improvement with the highest need for improvement. In each focus group, 3-5 women with endometriosis will participate. During these focus groups, participants are motivated to discuss the reason that the specific care aspect was previously identified as a target for improvement. Participants are subsequently asked to brainstorm on how these targets for improvement can be optimized. Focus group meetings will be organized until saturation occurs, which means that no new information is provided in the last focus group meeting. When saturation is anticipated, 1 more focus group will be organized to confirm saturation. All focus groups will be audiotape-recorded and transcribed verbatim. Analysis will be performed by independent researchers using sensitizing concepts with a modified grounded theory approach. This approach aids researchers to build themes from the data based on the concept themes that emerged from the study questions [20,21].

\section{Building the Improvement Plan}

An improvement plan will be drafted by the researchers using the input received from the focus groups. Feasibility is considered when drafting the improvement plan. When necessary, experts from the clinic (i.e., members of the multidisciplinary endometriosis team, residents, and nurses) will be consulted on their ideas in a focus group. Also, the Dutch endometriosis patient organization (Endometriose Stichting) will be invited to reflect on the improvement plan. Thereafter, it will be evaluated by representatives of the professional association on whether it truly aims to enhance the targets for improvement as found in the previous steps.

\section{Implementing Improvement}

The improvement plan will be presented to the team of the involved endometriosis clinic. Since part of the team was already involved in drafting the improvement plan, a high uptake of the improvement plan is expected. Monthly updates and reminders will be sent to the team, and monthly discussion groups will be organized to evaluate current improvements and to identify barriers for improvement.

\section{Evaluating the Improvement}

One and a half year after the implementation of the improvement plan, a new evaluation of patient-centredness of endometriosis care will be performed. Again, the ECQ will be distributed among women who visited the endometriosis care clinic in the previous 6 months. The results of the ECQ will be compared to the results found in step 1 .

\section{Participants}

All women with endometriosis, diagnosed by physical examination, radiologic investigation, or surgically, are eligible for inclusion for steps 1,2 , and 5 . Women need to be $>18$ years of age and Dutch-speaking. Women can participate in $>1$ step, but this is not necessary. For the focus groups (step 2), we aim to include women from various backgrounds in order to obtain a broad perspective of patients' ideas and opinions. Diverseness will be obtained by including women from different ethnic backgrounds, different educational levels, and from the different groups that were previously identified as determinants of patient-centred endometriosis care [22]. For steps 1 and 5, eligible women who visited the evaluated endometriosis clinic are asked to participate.

\section{Sample Size}

For step 1 and step 5, we will contact all women with endometriosis who had an appointment in the endometriosis clinic in the preceding 6 months. After the initial invitation to participate, we will send a reminder to all non-responders. We aim for a response rate of at least $50 \%$. Previous published group sizes per investigated clinic included $76-216$ persons $[15,16]$. Focus groups will be organized with 3-5 participants per focus groups and will be organized until saturation occurs. Saturation is achieved when the researchers do not find any new information in the focus groups [23]. Recent research involving endometriosis patients has shown that saturation can be achieved after attending 3-4 focus group meetings [24]. Therefore, we expect to organize 3-4 focus group meetings in order to achieve saturation.

\section{Analysis}

The results of the ECQ will be analyzed using the manual [15]. Targets for improvement are identified as care aspects that are important (mean important score $>$ overall important score) and have a poor performance (PNP $>15)$ according to the scoring in the ECQ. Comparison of the first and second usages of the ECQ will be performed by the independent $t$ test or Mann-Whitney $\mathrm{U}$ test depending on the distribution of the outcomes.

\section{Discussion}

This study aimed to improve endometriosis care by identifying targets for improvement of patient-centredness of endometriosis care, and subsequently by the de- 
velopment of an improvement plan, which is created with the input from both patients and health-care providers. By involving patients in focus group meetings, the targets for improvement can be explored more in depth, and specific improvements can be formulated using qualitative research until saturation occurs. Involving the healthcare providers is essential in achieving a successful implementation of changes in care [25].

As quality of endometriosis care might be correlated with QoL [26] and QoL is impaired in women with endometriosis with a large psychological, social, and sexual impact [27-29], it is of utmost importance to optimize quality of endometriosis care. In the past, women with endometriosis reported that the following aspects of care could be improved: (1) health-care providers' lack of essential knowledge, (2) inconsistent advice and, (3) the feeling of not being taken seriously [16, 30-33]. Moreover, 3 corresponding targets for improvement of patient-centred endometriosis care were found in 4 endometriosis clinics in Belgium and the Netherlands: (i) receiving practical information and advice on self-care after surgery, (ii) receiving information on pain medication, and (iii) personal follow-up by the treating physician $[15,16]$. These targets for improvement are acknowledged by health-care providers, and a desire for individualized, multidisciplinary care is presented [12, $30,34]$. Other targets for improvement reported by women with endometriosis might be focused on personalized medicine with attention for possible alternative therapies [35-37] or alternative methods for the advised treatment [38].

As a few years have passed since the last measurement of patient-centredness using the ECQ, the aforementioned targets for improvement are not necessarily still valid and reliable $[15,16]$. Even though no projects have been started to improve patient-centredness of endometriosis care, changes not noted in care might have occurred. Furthermore, patients' views and perspectives on importance may also have changed over time. Moreover, targets for improvement might differ from previous ECQ investigations as the inclusion criteria for the current study are different from the previous measurement as in the previous study solely women with surgically treated endometriosis were included. A new baseline measurement will therefore be performed for this study in step 1 . In today's practice, women with endometriosis are treated after diagnosis is made with certainty by physical examination, advanced radiologic examination, or surgery. Even though most current studies still aim to include surgically diagnosed patients only, we will include all diag- nosed endometriosis patients in order to mimic current practice and increase the sample size.

In the past, research in fertility care showed that presenting the health-care providers with targets of improvement alone is not sufficient enough, even though healthcare providers realize the importance of improving care [39]. By including not only patients but also health-care providers in the improvement of endometriosis care, this project aims to optimize the feeling of involvement and responsibility to improve endometriosis care among health-care providers [25]. Per target for improving a specific, concrete, and feasible improvement plan should be constituted carefully. Implementation of an improvement plan will be a continuous process using the model according to Wensing and Grol [25]. If possible, pitfalls and barriers in the implementations should be apprehended beforehand. Whether this project truly improves patient-centredness of endometriosis care will be investigated in step 5 .

In the Netherlands, the Dutch Society of Obstetrics and Gynaecology (NVOG) accredits all hospitals for quality of care by performing audits. In 2020, the Dutch Society of Obstetrics and Gynaecology special interest group "endometriosis" has drafted up a proposal for all endometriosis clinics in the Netherlands. Their aim is to centralize endometriosis care in order to improve quality of endometriosis care [40]. Both the accreditation and the centralization run separately from the current study. However, if effective, our proposed method of improving endometriosis care could be adapted as a tool in the process of future audits in the Netherlands.

Even though the ECQ has been used to investigate patient-centredness, the ECQ has never been used to structurally improve patient-centredness of endometriosis care. If the current study prooves to be able to successfully improve patient-centredness of endometriosis care, this method may be used, after further validation, as instrument for auditing value-based health care. The aim is to compose an easily accessible improvement strategy for health-care providers to be used to enhance patient-centredness of endometriosis care. When proven successful, this method could also be used by health-care providers in the field of other chronic diseases (i.e., chronic obstructive pulmonary disease and rheumatoid arthritis), although a different tool should be used to investigate patient-centredness as the ECQ is specifically developed and validated for the evaluation of endometriosis care.

A possible limitation of the study is that the drafted improvement plan is clinic specific and can possibly not be extrapolated to other endometriosis clinics. In order to aim 
to improve patient-centred endometriosis care elsewhere, the complete study protocol should be performed. Another limitation might be that we include Dutch-speaking women only, as the spoken language during the focus groups is Dutch. This implies that we currently miss the perspective of women with a non-Dutch background although it is not excluded that they might also be represented in the Dutchspeaking population. A strength of the study is to optimize the chances of succeeding in a durable improvement, by involving both patients and health-care providers, and by striving to improve the care in a feasible way.

\section{Conclusion}

This study protocol aimed to validate a tool to improve patient-centred endometriosis care by investigating underlying causes for poor performance on patient-centred care. Both patients and health-care providers will be involved in drafting the improvement plan. By making the health-care providers responsible for improving care, chance of succeeding is optimized. Whether this improvement strategy is successful will be investigated after implementation of the improvement plan.

\section{Statement of Ethics}

Ethical approval was granted by the Institutional Review Board of the Amsterdam UMC, Vrije Universiteit Amsterdam (ref number 2018.438). Consent to participate was not applicable for this study protocol. In the final study, participants will sign informed consent before participating in focus groups. Consent for the questionnaires will be based on implied consent when participants complete the questionnaires.

\section{Conflict of Interest Statement}

V.M. and C.B. report grants from Guerbet, grants from Merck, and grants form Ferring outside the submitted work. This study was investigator-initiated, and the execution of this study will be funded through an unrestricted grant by Merck.

\section{Funding Sources}

This study was investigator-initiated, and the execution of this study will be funded through an unrestricted grant by Merck.

\section{Author Contributions}

A.S., L.H. and V.M. initiated the design of the study. All authors were involved in the revision of the design of this study. A.S. drafted the manuscript. All authors critically revised and approved the final version of the manuscript.

\section{Data Availability Statement}

Not applicable.

\section{References}

1 Zondervan KT, Becker CM, Missmer SA. Endometriosis. N Engl J Med. 2020;382(13): 1244-56.

2 Dunselman GA, Vermeulen N, Becker C, Calhaz-Jorge C, D'Hooghe T, De Bie B, et al. ESHRE guideline: management of women with endometriosis. Hum Reprod. 2014;29(3): 400-12.

3 Simoens S, Dunselman G, Dirksen C, Hummelshoj L, Bokor A, Brandes I, et al. The burden of endometriosis: costs and quality of life of women with endometriosis and treated in referral centres. Hum Reprod. 2012;27(5): 1292-9.

4 Huntington A, Gilmour JA. A life shaped by pain: women and endometriosis. J Clin Nurs. 2005;14(9):1124-32.

5 Jones G, Jenkinson C, Kennedy S. The impact of endometriosis upon quality of life: a qualitative analysis. J Psychosom Obstet Gynaecol. 2004;25(2):123-33.
6 De Graaff AA, D'Hooghe TM, Dunselman GA, Dirksen CD, Hummelshoj L; WERF EndoCost Consortium, et al. The significant effect of endometriosis on physical, mental and social wellbeing: results from an international cross-sectional survey. Hum Reprod. 2013; 28(10):2677-85.

7 Facchin F, Barbara G, Saita E, Mosconi P, Roberto A, Fedele L, et al. Impact of endometriosis on quality of life and mental health: pelvic pain makes the difference. J Psychosom Obstet Gynaecol. 2015;36(4):135-41.

8 Friedl F, Riedl D, Fessler S, Wildt L, Walter M, Richter R, et al. Impact of endometriosis on quality of life, anxiety, and depression: an Austrian perspective. Arch Gynecol Obstet. 2015;292(6):1393-9.
9 Marinho MCP, Magalhaes TF, Fernandes LFC, Augusto KL, Brilhante AVM, Bezerra LRPS. Quality of life in women with endometriosis: an integrative review. J Womens Health. 2018;27(3):399-408.

10 Saunders PTK, Horne AW. Endometriosis: etiology, pathobiology, and therapeutic prospects. Cell. 2021;184(11):2807-24.

11 Vercellini P, Viganò P, Somigliana E, Fedele L. Endometriosis: pathogenesis and treatment. Nat Rev Endocrinol. 2014;10(5):26175.

12 Agarwal SK, Foster WG, Groessl EJ. Rethinking endometriosis care: applying the chronic care model via a multidisciplinary program for the care of women with endometriosis. Int J Womens Health. 2019;11:405-10.

13 World Health Organization. Quality of care: a process for making strategic choices in health systems. WHO Press; 2006.
Improving Patient-Centredness in Endometriosis Care: A Study Protocol 
14 Dancet EA, Ameye L, Sermeus W, Welkenhuysen M, Nelen WL, Tully L, et al. The ENDOCARE questionnaire (ECQ): a valid and reliable instrument to measure the patientcenteredness of endometriosis care in Europe. Hum Reprod. 2011;26(11):2988-99.

15 Dancet EA, Apers S, Kluivers KB, Kremer JA, Sermeus W, Devriendt C, et al. The ENDOCARE questionnaire guides European endometriosis clinics to improve the patient-centeredness of their care. Hum Reprod. 2012; 27(11):3168-78.

16 Schreurs AMF, van Hoefen Wijsard M, Dancet EAF, Apers S, Kuchenbecker WKH, van de Ven PM, et al. Towards more patientcentred endometriosis care: a cross-sectional survey using the ENDOCARE questionnaire. Hum Reprod Open. 2020;2020(3):hoaa029.

17 Geukens EI, Apers S, Meuleman C, D'Hooghe TM, Dancet EAF. Patient-centeredness and endometriosis: definition, measurement, and current status. Best Pract Res Clin Obstet Gynaecol. 2018;50:11-7.

18 Wisdom J, Creswell JW. Mixed methods: integrating quantitative and qualitative data collection and analysis while studying patient-centered medical home models. Rockville, MD: Agency for Healthcare Research and Quality; 2013. AHRQ Publication No. 130028-EF.

19 van Campen C, Sixma HJ, Kerssens JJ, Peters L, Rasker JJ. Assessing patients' priorities and perceptions of the quality of health care: the development of the QUOTE-rheumatic-patients instrument. Br J Rheumatol. 1998; 37(4):362-8.

20 Bowen GA. Grounded theory and sensitizing concepts. Int J Qual Methods. 2006;5(3):1223.

21 Braun VCV. Using thematic analysis in psychology. Qual Res Psychol. 2006;3(2):77-101

22 Schreurs AMF, Dancet EAF, Apers S, van Hoefen Wijsard M, Kuchenbecker WKH, van de Ven PM, et al. A systematic review and secondary analysis of two studies identifying de- mographic and medical characteristics determining patient-centeredness in endometriosis care as experienced by patients. Hum Reprod Open. 2020;2020(3):hoaa041.

23 Saunders B, Sim J, Kingstone T, Baker S, Waterfield J, Bartlam B, et al. Saturation in qualitative research: exploring its conceptualization and operationalization. Qual Quant. 2018;52(4):1893-907.

24 Metzemaekers J, Slotboom S, Sampat J, Vermolen P, Smeets MJGH, Elske van den Akkervan Marle $\mathrm{M}$, et al. Crossroad decisions in deep endometriosis treatment options: a qualitative study among patients. Fertil Steril. 2021;115(3):702-14.

25 Wensing M, Grol R. Implementatie: effectieve verbetering van de patiëntenzorg. Bohn Stafleu van Loghum; 2017. Vol. 7.

26 Apers S, Dancet EAF, Aarts JWM, Kluivers KB, D'Hooghe TM, Nelen WLDM. The association between experiences with patientcentred care and health-related quality of life in women with endometriosis. Reprod Biomed Online. 2018;36(2):197-205.

27 La Rosa VL, De Franciscis P, Barra F, Schiattarella A, Török P, Shah M, et al. Quality of life in women with endometriosis: a narrative overview. Minerva Med. 2020;111(1):68-78.

28 La Rosa VL, De Franciscis P, Barra F, Schiattarella A, Tropea A, Tesarik J, et al. Sexuality in women with endometriosis: a critical narrative review. Minerva Med. 2020;111(1):79-89.

29 Simoens S, Hummelshoj L, Dunselman G, Brandes I, Dirksen C, D'Hooghe T, et al. Endometriosis cost assessment (the EndoCost study): a cost-of-illness study protocol. Gynecol Obstet Invest. 2011;71(3):170-6.

30 Rowe HJ, Hammarberg K, Dwyer S, Camilleri R, Fisher JR. Improving clinical care for women with endometriosis: qualitative analysis of women's and health professionals' views. J Psychosom Obstet Gynaecol. 2021;42(3): 174-80.

31 Findeklee S, Radosa JC, Mothes A, Younes S, Schafhaupt S, Stotz L, et al. Patient satisfac- tion with personal patient care (PPC) in the inpatient treatment of endometriosis. Arch Gynecol Obstet. 2020;301(2):545-50.

32 Denny E. Women's experience of endometriosis. J Adv Nurs. 2004;46(6):641-8.

33 Lukas I, Kohl-Schwartz A, Geraedts K, Rauchfuss M, Wölfler MM, Häberlin F, et al. Satisfaction with medical support in women with endometriosis. PLoS One. 2018;13(11): e0208023.

34 Simonsen SM, Strømberg C, Zoffmann V, Hartwell D, Olesen ML. About me as a person not only the disease - piloting guided self-determination in an outpatient endometriosis setting. Scand J Caring Sci. 2020;34(4):101727.

35 Della Corte L, Noventa M, Ciebiera M, Magliarditi M, Sleiman Z, Karaman E, et al. Phytotherapy in endometriosis: an up-todate review. J Complement Integr Med. 2020; 17(3)

36 Huijs E, Nap A. The effects of nutrients on symptoms in women with endometriosis: a systematic review. Reprod Biomed Online. 2020;41(2):317-28

37 Xu Y, Zhao W, Li T, Zhao Y, Bu H, Song S. Effects of acupuncture for the treatment of endometriosis-related pain: a systematic review and meta-analysis. PLoS One. 2017; 12(10):e0186616

38 Garzon S, Laganà AS, Barra F, Casarin J, Cromi A, Raffaelli R, et al. Novel drug delivery methods for improving efficacy of endometriosis treatments. Expert Opin Drug Deliv. 2021;18(3):355-67.

39 Huppelschoten AG, Aarts JW, van Empel IW, Cohlen BJ, Kremer JA, Nelen WL. Feedback to professionals on patient-centered fertility care is insufficient for improvement: a mixedmethod study. Fertil Steril. 2013;99(5):1419_ 27.

40 de Kok L, van Hanegem HN, van Kesteren P, Klinkert E, Maas J, Mijatovic V, et al. De ontwikkeling van endometrioseexpertisecentra in Nederland. NTOG. 2020;133(5):242-5. 